\title{
UTILIZAÇÃO DE FERRAMENTA DE ANALYTICS NO LAMINADOR DE TIRAS A QUENTE DE TUBARÃO*
}

\author{
Anderson Esteves Bragança ${ }^{1}$ \\ Camila Fernandes Rezende ${ }^{2}$ \\ Lavínia Misson Cordeiro ${ }^{3}$ \\ Márcio Adriano Ribeiro ${ }^{4}$
}

\section{Resumo}

Foi identificado na área da unidade técnica da laminação de tiras a quente uma necessidade de cruzamento de dados de diversos sistemas de nível 1 (Instrumentação), nível 2 (Automação) e nível 3 (TI), para análise do processo. Todo esse processo de extração e formatação dos dados levava dias, com necessidade de suporte da equipe de $\mathrm{TI}$, ou às vezes nem era possível devido à complexidade de cruzamento de informações. A ideia era prover uma ferramenta capaz de acessar diferentes bases de dados permitindo a integração dessas informações de forma simplificada e ágil. Após avaliação de ferramentas disponíveis no mercado (Ferramentas de Analytics e BI) a opção foi por uma que trouxe: a integração dessas bases, redução do tempo gasto na preparação de indicadores e um maior tempo disponível para investigação e solução de problemas por parte dos especialistas. A conclusão do projeto foi uma unidade técnica mais produtiva e autônoma, com uma menor dependência da equipe de informática e automação.

Palavras-chave: Integração de dados; Ferramentas de analytics; QlikView; Indicadores de processo.

\section{Abstract}

\section{WORKING WITH ANALYTIC TOOL IN THE HOT STRIP MILL}

To analyze the Hot Strip Mill (HSM) process, the technical division requires several data query from different sources, as Level 1 (Instrumentation data), Level 2 (Automation data) and Level 3 (IT data). The whole extraction process and data formatting demanded excessive time, apart from the need of a full-time IT specialist to support the report building by the technical staff, making it unfeasible depending on its complexity. The idea of the project was to get a software that would be capable to access different databases at the same time, allowing a perfect integration among them in an easier and agiler way. Based on a research in the market it has been decided for a tool that could provide: database integration, lower time required to build key process indicators and an increased available time for investigation and problem solving by the process specialists. The project output is a more productive and autonomous technical division, with less dependence on the IT and Automation team.

Keywords: Data Integration; Analytics tools; QlikView; KPI's.

1 Bacharel em Ciência da Computação, Analista de Sistemas, departamento de TI, ArcelorMittal Tubarão, Serra, Espírito Santo, Brasil.

2 Bacharel em Ciência da Computação, Analista de Sistemas, departamento de TA, ArcelorMittal Tubarão, Serra, Espírito Santo, Brasil.

3 Engenheira Metalurgista, Especialista de Processo, Unidade Técnica de Tiras a Quente, ArcelorMittal Tubarão, Serra, Espírito Santo, Brasil.

4 Bacharel em Sistema de Informação, Analista de Sistemas, departamento de TI, ArcelorMittal Tubarão, Serra, Espírito Santo, Brasil. 


\section{INTRODUÇÃO}

Com capacidade anual de $4 \mathrm{Mt}$, o Laminador de Tiras a Quente (LTQ) da ArcelorMittal Tubarão precisa atender as especificações dos clientes e requisitos de qualidade, visando sempre redução de retrabalhos e custo. Esse processo é complexo e possui inúmeras variáveis de controle, com necessidade de acompanhamento através de KPl's (Key Performance Indicator).

A unidade técnica do LTQ sempre teve grande necessidade de cruzamento de informações provenientes dos diversos sistemas de controle da linha, do nível de instrumentação/automação até o de controle de processo, sempre com o objetivo de elaboração de indicadores que permitissem o acompanhamento e melhorias da performance e qualidade.

Até então esse trabalho era realizado pelos especialistas do laminador, com o auxílio das equipes de Automação e TI, utilizando planilhas acessando diretamente as respectivas bases de dados desses sistemas. Toda a preparação de relatórios demandava grande esforço prévio para que as informações estivessem disponíveis e correlacionadas para então dar início ao estudo necessário. Havia uma grande inversão de tempo gasto, causando uma enorme improdutividade, isto é, muito tempo gasto na preparação e cruzamento de dados e pouco tempo investido em experimentação e análise para melhorias de processo.

Além do mais, esses relatórios eram individualizados, isto é, cada um dependia de uma solução específica e, dependendo da sua complexidade, nem era possível sua elaboração. Como fruto dessa individualização, o compartilhamento desses relatórios entre a equipe era baixo, provocando retrabalhos desnecessários quando no uso de informações comuns e até mesmo indisponibilidade do KPI na ausência da pessoa que o havia feito.

O objetivo do projeto era proporcionar aos usuários uma forma mais produtiva de trabalho através de uma ferramenta capaz de cruzar diversas fontes de dados de maneira transparente e confiável. Uma solução que minimizasse a dependência das equipes de Tl/Automação e aumentasse a produtividade dos especialistas da área.

A finalidade era entregar um modelo de dados único, onde as informações de Instrumentação, Automação e TI já estariam cruzadas, dando a possibilidade aos usuários de "experimentar" diversas visões de um mesmo indicador de forma selfservice, auxiliando-os nas tomadas de decisões.

\section{MATERIAIS E MÉTODOS}

Com o objetivo mencionado acima, em meados de 2015 foi pesquisado junto ao Gartner [1], instituição independente que trabalha com pesquisa e consultoria na área de tecnologia da informação, ferramentas e fornecedores disponíveis que atendessem aos requisitos do projeto. Foi então apresentada à nova plataforma de desenvolvimento de BI (Business Intelligence) e Analytics, e dentro desse modelo de ferramentas, os seus principais players de mercado. 
Segundo o Gartner, as ferramentas apresentadas no quadrante superior direito (Leaders), Figura 1, são as mais bem avaliadas. Dentre elas, duas foram selecionadas para prova de conceito (POC): a primeira, Business Objects (BO) da SAP, é atualmente utilizada na planta de Dunkerque também na área do LTQ, a segunda, QlikView da Qlik, havia sido bem avaliada em 2013 na planta de Tubarão para atender a uma outra demanda que não evoluiu.

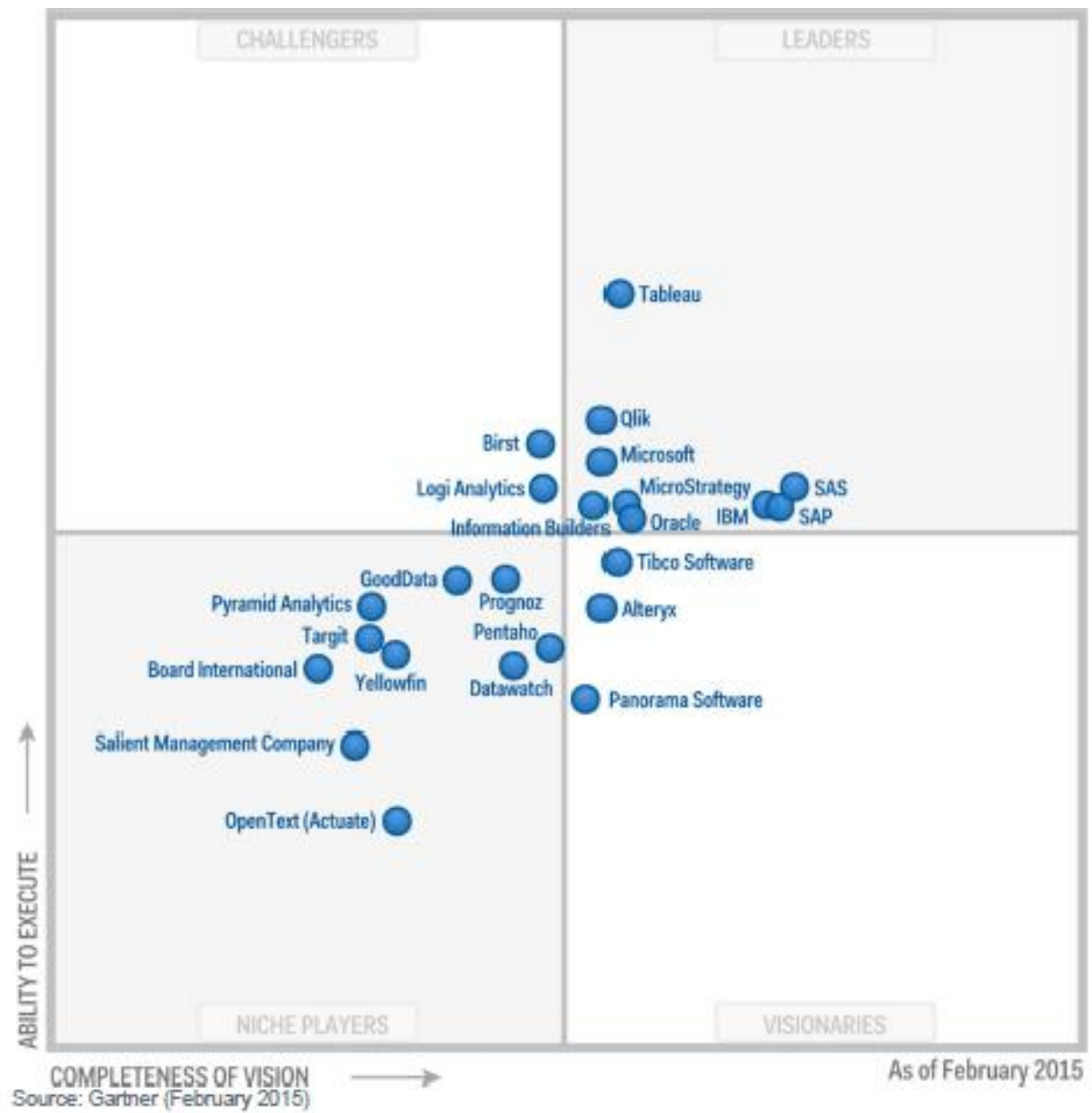

Figura 1: Quadrante Mágico para Ferramentas da Plataforma BI e Analytics - Fev 2015

Depois de muita pesquisa, avaliação, experimentação e discussões internas entre a área de Tl/Automação e os especialistas do processo de laminação, optou-se pelo QlikView. As principais vantagens identificadas na ferramenta escolhida foram:

- Trabalha em memória;

- Reúne todas as etapas de $\mathrm{BI}$ em uma única ferramenta (extração, transformação e apresentação);

- Acessa e mescla diversas fontes de dados (BD, planilhas,.txt);

- Baixo tempo e esforço de implementação;

- De fácil utilização pelo usuário final.

Em janeiro de 2016 iniciou-se o projeto de substituição de todo volume de planilhas de controle existente, por uma solução que fosse mais produtiva, robusta e corporativa. Foram identificados os principais KPl's que deveriam ser apresentados em uma aplicação corporativa, bem como as tabelas que deveriam fazer parte do 
modelo e contemplasse os assuntos: LTQ, Oficina de Cilindros e Paradas de Linha. Ao todo foram relacionadas 32 tabelas de nível 3, 12 de nível 2 e 3 de nível 1.

Durante a implantação do projeto ficou definido que, das 47 tabelas selecionadas, todas as informações seriam levadas para o modelo. Isso facilitaria consultas futuras que eventualmente poderiam necessitar dessas variáveis. $E$ também que os dados seriam levados para o ambiente de $\mathrm{BI}$ o mais próximo possível do modelo relacional, facilitando a implementação do projeto.

\section{RESULTADOS E DISCUSSÃO}

Após o levantamento dos principais indicadores da linha e de todas as variáveis de controle, foi elaborado um modelo integrado que fosse possível cruzar dados de toda a linha de laminação e de todos os sistemas envolvidos no processo (nível 1, nível 2 e nível 3). Esse modelo conta com aproximadamente 5.000 variáveis de controle totalmente correlacionadas entre si de forma transparente para o usuário. Na Figura 2 é possível observar de forma simplificada o fluxo da informação.

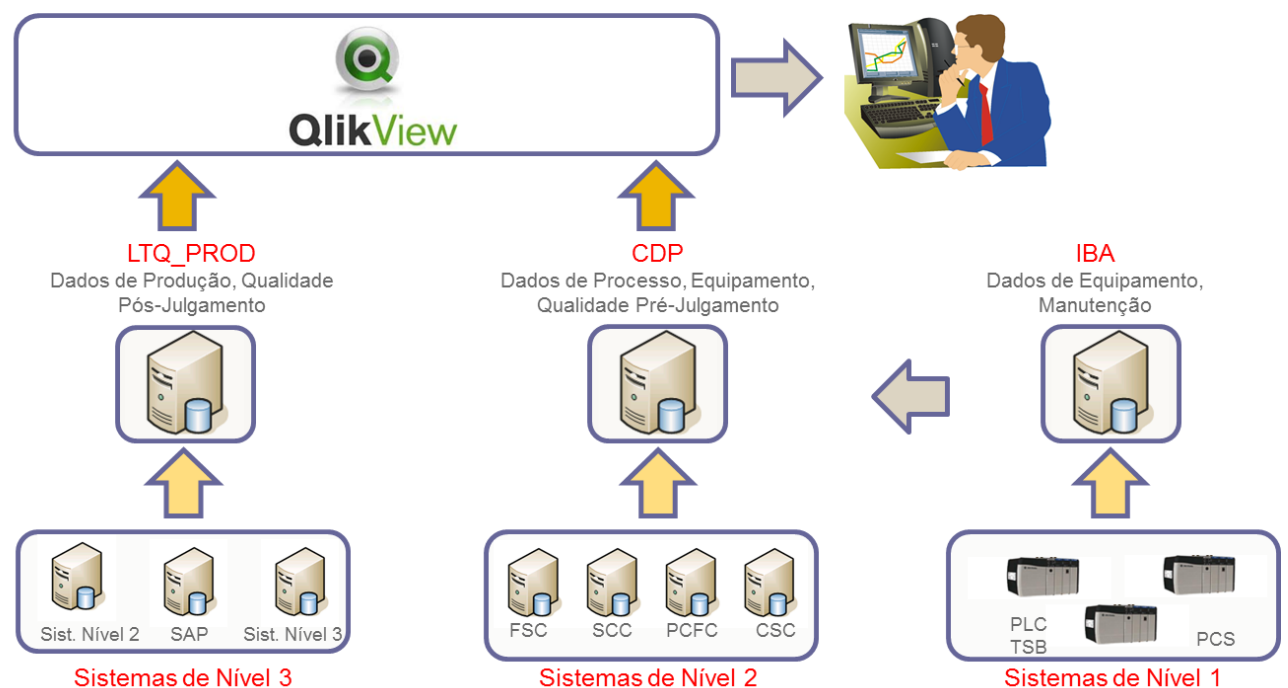

Figura 2: Esquema do fluxo da informação

Ao final do projeto foi entregue uma aplicação corporativa, com atualização diária, contendo 70 gráficos e tabelas abrangendo todos os setores do laminador e oficina de cilindros, além de informações sobre paradas e produtividade, Figura 3. A aplicação é dinâmica, ou seja, filtros podem ser aplicados de acordo com a necessidade do usuário e, após um ano da implantação já contempla 120 gráficos. Nas atividades de rotina, cada especialista constrói seus indicadores de interesse, disponíveis através das aplicações individuais. Caso haja necessidade, gráficos ou tabelas particulares podem ser transferidos para a aplicação corporativa pela equipe de TI/TA, sendo então visualizados por todo o grupo. 


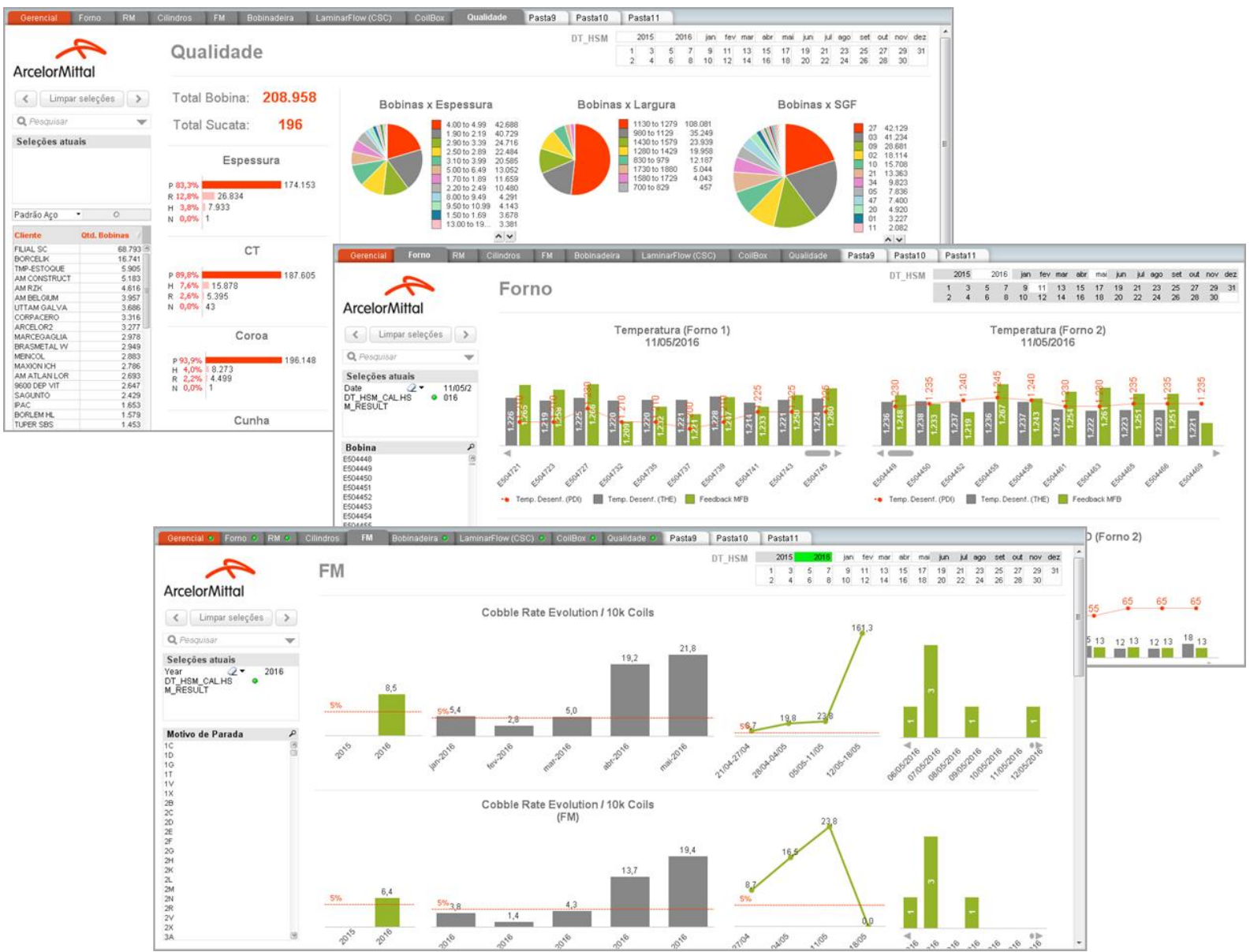

Figura 3: Exemplo de algumas telas da aplicação corporativa

De todos os dados disponíveis, apenas 500 variáveis foram utilizadas na construção da aplicação inicial. Considerando as aplicações individuais, estima-se que cerca de 1.000 variáveis são utilizadas. Esse total não ultrapassa $20 \%$ das informações disponíveis. Pela facilidade de inclusão de novas variáveis, inclusive seu histórico, em novos projetos deste tipo, a estratégia será exportar para o ambiente de BI somente o que for necessário aos usuários, à medida que forem demandados. Essa estratégia traz benefícios como: um ambiente mais rápido e fácil de ser utilizado, ainda atendendo aos requisitos do usuário em sua plenitude.

Diferente do que foi previsto na fase inicial, o modelo relacional não foi capaz de entregar um ambiente adequado e autônomo para criação de relatórios e indicadores pelo usuário final. Foram necessárias transformações para o modelo de $\mathrm{BI}$, ou seja, através de funções disponibilizadas pela ferramenta, foi possível criar associações que o modelo relacional não fazia, sempre respeitando as regras de negócio.

Além do modelo de dados integrado, foi desenvolvido também um relatório diário contendo os principais KPl's da linha de laminação. Esse documento é gerado e enviado automaticamente para todos os especialistas e gerentes envolvidos no processo com o fechamento da produção do dia anterior.

O projeto foi concluído em junho de 2016 e desde então tem auxiliado a equipe da unidade técnica do LTQ em suas diversas análises e melhorias no processo de 
laminação da ArcelorMittal Tubarão, tornando realidade seus anseios por mais autonomia e velocidade para gerar e consumir os dados do processo.

\section{CONCLUSÃO}

A ferramenta selecionada atendeu os requisitos do projeto, possibilitando a entrega para equipe do LTQ de um modelo de dados integrado, ágil e de fácil consulta.

Através de uma aplicação corporativa, os indicadores de processo são visualizados por todos da mesma forma e, a utilização de filtros possibilita que o usuário avalie 0 resultado somente do material que lhe interessa.

A utilização da ferramenta trouxe independência aos especialistas da área, aumentando a produtividade da unidade técnica. As planilhas Excel foram abandonadas para fins de exploração de dados e estima-se um ganho de 15 horas semanais que antes eram utilizados na preparação das informações.

Um relatório diário em PDF é enviado automaticamente com os principais KPI's do LTQ e abrange um público grande na usina, visto que não se faz necessário o licenciamento do produto QlikView.

O sucesso do projeto no LTQ foi grande, tem data para ser implantado na área de Metalurgia/Qualidade, e já possui demanda para acontecer nas demais unidades técnicas.

Em função do baixo percentual de variáveis utilizadas, nas próximas implantações as informações serão levadas conforme necessidade, melhorando o desempenho e agilidade do sistema.

\section{REFERÊNCIAS}

$1 \quad$ Link de acesso a assuntos relativos a BI e Analytics dentro do Gartner:

http://www.gartner.com/technology/topics/data-analytics.jsp. 